\title{
Atomic pectroscopy 1 \\ Rapid Elemental Determination and Discrimination of Coins Using Laser-induced Breakdown Spectroscopy
}

\author{
Haochen Peng, ${ }^{\text {a,b }}$ Yuzhu Liu, ${ }^{\text {a,b,* }}$ Ying Chen, ${ }^{\text {a,b }}$ Xu Lu, ${ }^{\text {a,b }}$ Guoqing Chen, ${ }^{\text {a,b }}$ and Yu Chen ${ }^{\text {a,b }}$ \\ a Jiangsu Key Laboratory for Optoelectronic Detection of Atmosphere and Ocean, Nanjing University of Information Science \& Technology, Nanjing 210044 , \\ P. R. China \\ ${ }^{\mathrm{b}}$ Jiangsu Collaborative Innovation Center on Atmospheric Environment and Equipment Technology (CICAEET), Nanjing 210044, P. R. China
}

Received: April 02, 2021; Revised: May 16, 2021; Accepted: May 16, 2021; Available online: May 18, 2021.

DOI: $10.46770 / A S .2021 .128$

ABSTRACT: Damaged coins can be identified effectively via spectral analysis based on LIBS, which is of great significance for coin recycling. This paper takes the Renminbi (RMB), the Chinese currency, as the example, including the denominations of YI FEN, ER FEN, WU FEN, YI JIAO, WU JIAO and YI YUAN. Some characteristic lines of $\mathrm{Mg}, \mathrm{Al}, \mathrm{Fe}, \mathrm{Cr}, \mathrm{Cu}, \mathrm{Sn}, \mathrm{Ni}, \mathrm{Na}$ and $\mathrm{Ca}$ were observed in the spectra, as well as the molecular bands of AlO. Principal component analysis (PCA) was used to reduce the dimension of the spectra of the different RMB coins. The samples after dimension reduction are classified by k-Nearest Neighbors (KNN), and 4 categories were obtained with a classification accuracy of $100 \%$. Further, new spectra of different denomination RMB coins were added to the original data for the same analysis. The results are in good agreement which shows the potential of the combination of LIBS, PCA and KNN for the analysis and identification of different coins.

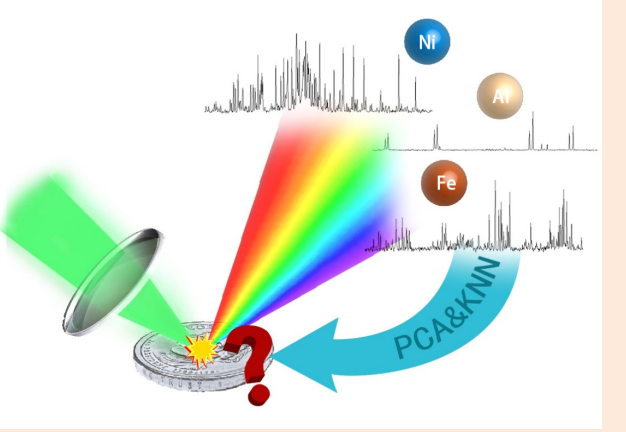

\section{INTRODUCTION}

The use of coins for payment of goods was introduced around the 6th century BCE. ${ }^{1,2}$ In ancient Rome ${ }^{2}$ and ancient China ${ }^{1}$ coins were once made of copper alloy. With the developments in commerce over time, coins have been continuously circulated as a kind of currency. ${ }^{3}$ At the same time, the technology of coin casting has gradually improved, and the material used for making coins has also changed. ${ }^{4}$ In modern times, the issue and use of coins/paper money and their circulation are closely related to people's lives, playing an important role in the promotion of economic development and contributing to the completion of transactions and for accounting.

In the circulation of coins, damage is inevitable..$^{5}$ In general, worn coins are recycled by banks for which they first need to establish financial statistics. After classification, these coins will be recast into new ones to enter the market. However, it is often difficult to distinguish the denomination of coin due to serious wear and tear, which increases the difficulty of replacing such coins for subsequent recasting. This problem is also encountered with ancient coins found in archaeological digs which are often worn and damaged coins ${ }^{6}$ and prove difficult to determine their category and dynasty.

Due to the similar size and color, many broken coins cannot be judged directly and rapidly by the naked eye. To identify worn and damaged coins, laser-induced breakdown spectroscopy (LIBS) is used to detect the elements of the coins. In addition, in order to recognize a large number of coins, it is necessary to classify them according to the different alloys. The spectral information of coins is dispersed in the principal component space by means of dimension reduction with principal component analysis (PCA). The thought of classification is to cluster the samples in the principal components space. Coins of the same material will gather in one area. Based on K-Nearest Neighbors (KNN), coins of different denominations can be classified. For coin detection work in a bank, this method can be a quick means to analyze the types of coins and reduces the time and economical cost involved. 
LIBS is a kind of spectral measurement method which can quickly extract the spectrum of substances. ${ }^{7-20}$ Compared to other conventional techniques such as X-ray fluorescence, LIBS requires minimum sample size, non-contact analysis, total elemental analysis, and rapid analysis in real-time..$^{21,22}$ It can widely detect solid, ${ }^{11-13}$ liquid, ${ }^{17-18}$ and gases (smoke). ${ }^{19}$ The size of ablation is in micron scale, which can be considered as industrial semi-destructive testing technology. At the same time, this method is not restrictive due to environmental conditions, which means that only relatively simple treatment needs to be carried out while preparing the samples. LIBS is widely used in medical, ${ }^{12}$ environmental, ${ }^{15,19}$ archaeological, ${ }^{22}$ and other fields because of its fast and real-time analysis.

PCA is an unsupervised analysis of classification (exploratory data analysis). High-dimensional data can be projected into a lower dimensional space by calculating the principal component, which means that only a few variables need to be used to divide the sample data of multiple variables into several groups. ${ }^{23}$ Several principal components to represent most of the information depends on the contribution rate of principal components; that is, the higher the contribution rate of a single principal component to the whole data, the more representative is the whole data. The variables between different samples are discrete wavelength values. The greater difference of variables between different samples, the more obvious the classification effect.

KNN is a kind of classification approach. The existing set of example data is used as the training set and a corresponding label (category) is given to each data. When a new piece of data is obtained without a label, the new piece of data is compared with each existing data. Then, the most similar pieces of data (the nearest neighbors) will be taken, and their labels will be checked. The top most similar $k$ pieces of data from the known dataset will be checked; this is where the $k$ comes from ( $k$ is an integer, which is usually less than 20.). Lastly, a majority of votes will be taken from the most similar pieces of $k$ data, and the majority is the new class that is assigned to the data asked to be classified. ${ }^{24-26}$ However, the previous LIBS study of coins is to analyze and compare ancient coins from different periods. ${ }^{22,27}$ The object of comparison are coins from different periods from the same area, and the differences in the elements found reflect their history, which is of great archaeological significance.

In this paper, different denominations of currency in circulation are compared and classified. A large number of coins with different denominations can be classified quickly and accurately. It is convenient to facilitate the subsequent coin recycling work. First, LIBS is used for the rapid detection to obtain the spectra of different coins and to distinguish the elements corresponding to the spectral peaks. Then, based on the dimension reduction by PCA, KNN is used as a means to classify the coins. New samples of different denominations are used to verify this classification method.

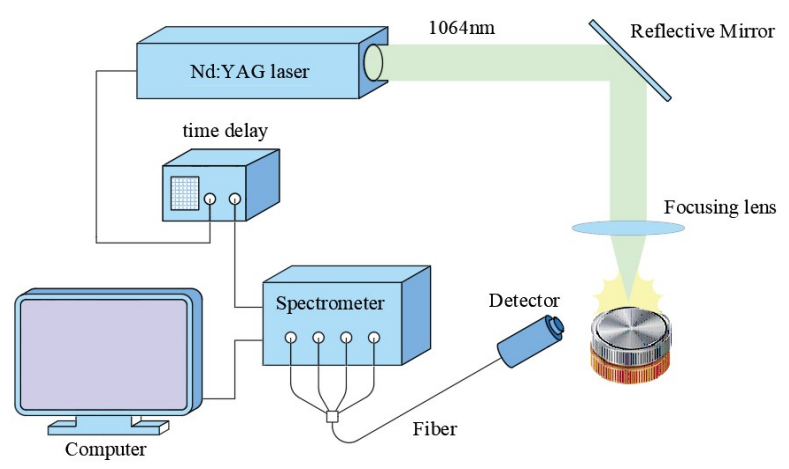

Fig. 1 Schematic diagram of the experimental setup.

\section{EXPERIMENTAL}

Experimental setup. The schematic diagram of the LIBS experiment is shown in Fig.1. To analyze all samples and excite all elements, a high-power Q-switch Nd:YAG (neodymium-doped yttrium aluminum garnet) laser with a fundamental wavelength of $1064 \mathrm{~nm}$ was used. One single pulse has $10 \mathrm{~ns}$ duration and $10 \mathrm{~Hz}$ operating with a pulse energy of $60 \mathrm{~mJ}$. The spot size value of the laser is about $7 \mathrm{~mm}$, and the irradiance is about $1.6 \times 10^{7} \mathrm{~W} / \mathrm{cm}^{2}$. The direction of the laser beam is changed through the mirror, and the sample is irradiated by a quartz convergent lens with a focal length of $5 \mathrm{~cm}$. The processed coin sample is placed on the platform and ablated into the plasma by laser. The radiation of the plasma is collected directly by the optical fiber. Since the laser can only interact with the small area of the sample surface at a time, the coin will be moved so that most of the surface of the coin has a chance to interact with the laser. The optical signal is received by the detector and then enters the LIBS spectrometer (AvaSpecULS2048-4Channel-usb2.0, Avantes) ${ }^{28}$ through the coupled fiber bundle. The spectral window of the spectrometer ranges from 200 $\mathrm{nm}$ to $890 \mathrm{~nm}$ and is divided into four channels. The spectral resolution of the spectrometer is about $0.1 \mathrm{~nm}$. To synchronize the detection part with the Nd:YAG laser, a time delay device between the laser and the spectrometer was set. To achieve better spectral resolution, the delay was set at $1.5 \mu \mathrm{s}^{29}$ The entire surface of the coin is sampled by raster mode. 600 samples of each coin were collected, and some samples with better spectral characteristics were selected for processing.

Sample preparation. Six kinds of coins were purchased online and used in the experiment, including YI FEN, ER FEN, WU FEN,

Table 1. The Equivalence of the Six Coins

\begin{tabular}{lcc}
\hline \multicolumn{1}{c}{ Symbol } & RMB coin & Equivalent coin \\
\hline A & YI FEN & 1 cent \\
B & ER FEN & 2 cents \\
C & WU FEN & 5 cents \\
D & YI JIAO & 10 cents \\
E & WU JIAO & 50 cents \\
F & YI YUAN & 100 cents \\
\hline
\end{tabular}


YI JIAO, WU JIAO, and YI YUAN. The equivalence relationship of the six coins is shown in Table 1. For simplicity, coins of different denominations will be represented by " $A$ " to " $F$ ". The cleanest looking coin from each kind of coin was chosen as the experimental sample. To simulate the real situation, these coins were simply cleaned and wiped with alcohol and mirror paper. After alcohol evaporation, each coin was wiped again. After repeating the operation five times, it can be considered that the grease and stains on the coin surface have been removed. Among them, the YI FEN (A), WU FEN (C), YI JIAO (D), WU JIAO (E) and YI YUAN (F) coins were used for detection and classification, the ER FEN (B) coin was used for verification.

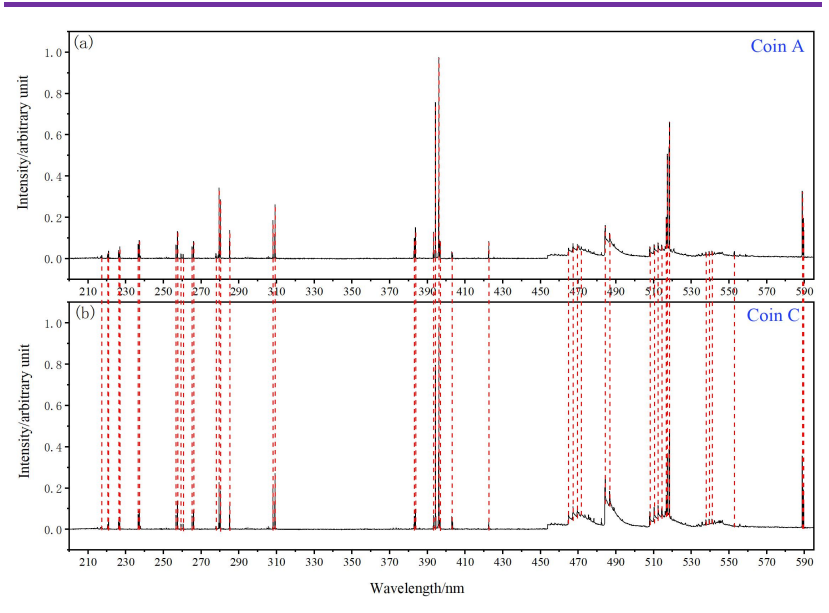

Fig. 2 Spectral range from 200 to $595 \mathrm{~nm}$ for coin A(a) and coin C(b). The lines in red are the emission lines characteristic of the elements present in the two coins.

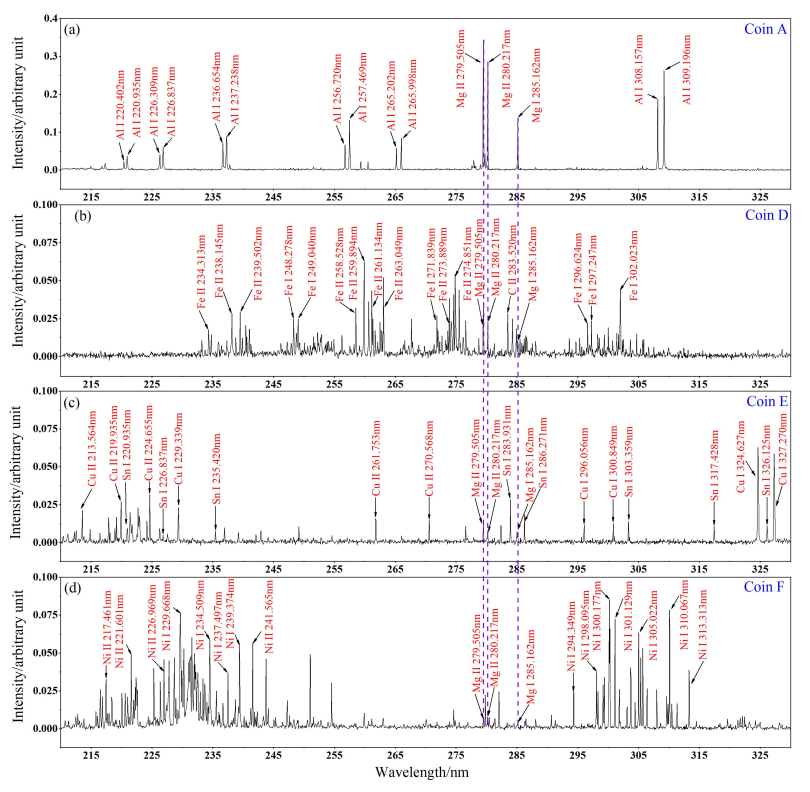

Fig. 3 Spectral range from 210 to $330 \mathrm{~nm}$ for coin A (a), coin D (b), coin E (c) and coin $\mathrm{F}(\mathrm{d})$. The lines in purple $(\mathrm{Mg})$ are the emission lines characteristic of the same elements present in the coins.
Table 2. Part of the Peak Wavelengths of Characteristic Spectral Lines of AlO Free Radical

\begin{tabular}{cccc}
\hline $\boldsymbol{\Delta} \boldsymbol{v}=+\mathbf{1}$ & $\boldsymbol{\Delta v}=\mathbf{0}$ & $\boldsymbol{\Delta} \boldsymbol{v}=\mathbf{- 1}$ & $\boldsymbol{\Delta} \boldsymbol{v}=\mathbf{- 2}$ \\
\hline $464.825 \mathrm{~nm}$ & $484.320 \mathrm{~nm}$ & $508.010 \mathrm{~nm}$ & $533.703 \mathrm{~nm}$ \\
$467.229 \mathrm{~nm}$ & $486.685 \mathrm{~nm}$ & $510.325 \mathrm{~nm}$ & $535.835 \mathrm{~nm}$ \\
$469.495 \mathrm{~nm}$ & $488.914 \mathrm{~nm}$ & $512.379 \mathrm{~nm}$ & $537.713 \mathrm{~nm}$ \\
$470.294 \mathrm{~nm}$ & $493.491 \mathrm{~nm}$ & $514.301 \mathrm{~nm}$ & $539.463 \mathrm{~nm}$ \\
$471.624 \mathrm{~nm}$ & $/$ & $516.092 \mathrm{~nm}$ & $540.960 \mathrm{~nm}$ \\
$473.617 \mathrm{~nm}$ & $/$ & $/$ & $542.205 \mathrm{~nm}$ \\
\hline
\end{tabular}

\section{RESULTS AND DISCUSSION}

\section{Rapid elemental determination in RMB coins}

Considering the characteristics of the LIBS technique, the spectral characteristics of the coin's surface are analyzed in this paper. In the detection part, coin A, C, D, E, F will be selected as the experimental objects for spectral analysis. Referring to the National Institute of Standard and Technology (NIST ${ }^{30}$ ) and related data, the result of the identification of elements can be well represented.

The spectral lines of different elements show the information contained in the coins. The spectral lines with the highest intensity were normalized according to all the spectral data of each sample. Fig. 2 shows the spectral range (200 to $595 \mathrm{~nm}$ ) for coin A and coin $\mathrm{C}$. In the identification process, it can easily be seen that the characteristic lines (marked by lines in red) of coin $\mathrm{A}$ and coin $\mathrm{C}$ are the same, which means that the elements contained in the two coins are consistent. The band from $595 \mathrm{~nm}$ to $890 \mathrm{~nm}$, which shows the characteristic spectrum of air elements obviously rather than the metal elements, will not be shown in the following presentation.

Therefore, in the later wavelength display, only the spectrum of coin A is shown, see Fig. 3 and Fig. S1-S3, which shows the relationship between wavelength and normalized intensity. The typical spectrum of coin $\mathrm{A}$ shows the $\mathrm{Al}^{31}$ and $\mathrm{Mg}$ lines. Meanwhile, the $\mathrm{AlO}$ free radical has characteristic emission bands in the range of 465 to $515 \mathrm{~nm}$. Coin D contains $\mathrm{Fe}$ and $\mathrm{Cr}$, coin $\mathrm{E}$ contains $\mathrm{Cu}$ and $\mathrm{Sn}$, and coin $\mathrm{F}$ contains Ni. Also, the $\mathrm{Mg}$ (lines in purple from Fig. 3), Ca (lines in blue from Fig. S1), and $\mathrm{Na}$ (lines in orange from Fig. S3) appear repeatedly in all coins, as the colored lines show.

As shown in Table 2, for the AlO free radical, there are four spectral bands (including $\Delta v=+1, \Delta v=0, \Delta v=-1, \Delta v=-2$ ). Each of the spectral bands with four to six peaks can be clearly observed in Fig. S2. Aluminum in $\mathrm{AlO}$ comes from the coin, while oxygen may be inherent in the coin itself, or it may come from the air. ${ }^{32}$

\section{Unsupervised analysis of five coins}

To simplify the identification and sorting of broken coins or ancient coins and to eliminate the mistakes caused by naked eye 

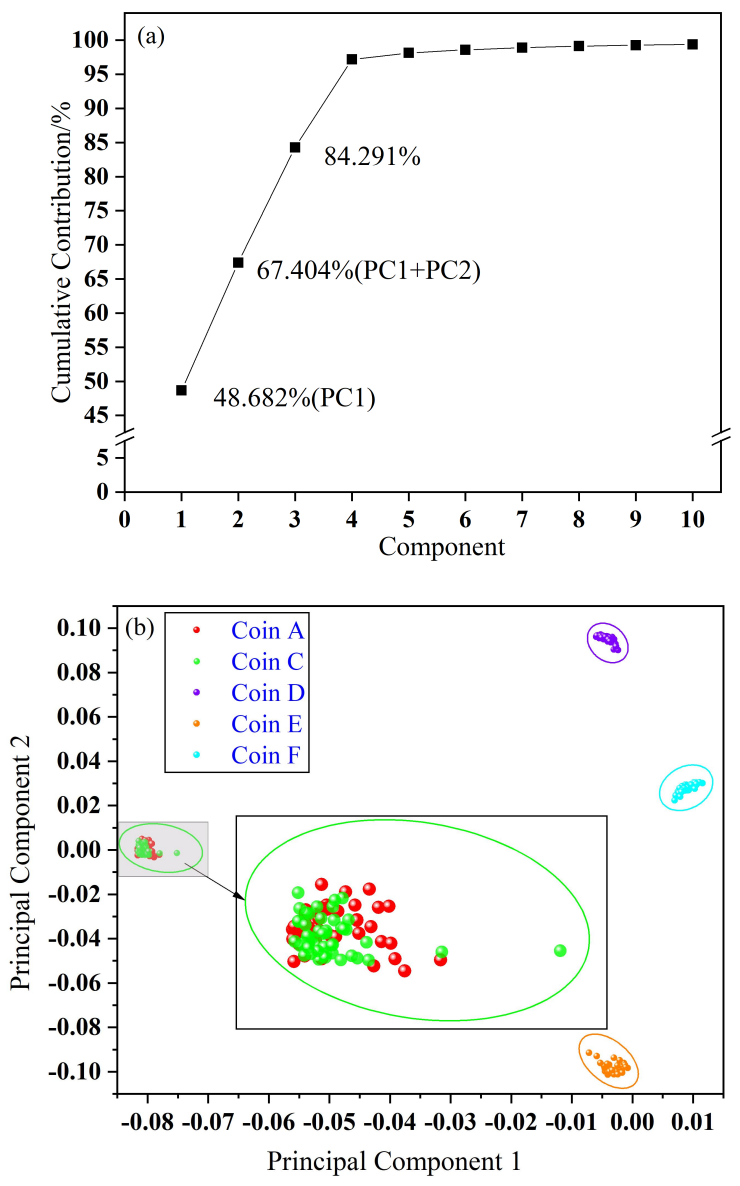

Fig. 4 (a) The cumulative contribution of the first 10 principal components from $215 \mathrm{~nm}$ to $240 \mathrm{~nm}$, (b) the result of PCA of five coins from $215 \mathrm{~nm}$ to $240 \mathrm{~nm}$.

identification, it is necessary to identify the elements of different coins with LIBS and reduce the dimension with PCA. The types and contents of elements in different coins are different, and their positions in the principal component space are also different. In the experiment, 600 samples were collected from each coin, and 50 samples with obvious spectral characteristics of each coin. There are two ways to analyze with PCA: (1) the whole wavelength range (from $200 \mathrm{~nm}$ to $890 \mathrm{~nm}$ ) and (2) the partial wavelength range. ${ }^{33}$

In the first way, the spectrum data from $200 \mathrm{~nm}$ to $890 \mathrm{~nm}$ will be selected. At the same time, to reflect the spectral information of the coin to a certain extent, there was no normalization. The contribution rates of the first principal component (PC1) and the second principal component (PC2) are $40.007 \%$ and $25.693 \%$, respectively. The cumulative contribution rate $(\mathrm{PC} 1+\mathrm{PC} 2)$ is $65.701 \%$, which can represent most of the spectral information of the samples. (Fig.S4a shows that the first 10 principal components add up). Fig. S4b shows the distribution of the samples in the space formed by $\mathrm{PC} 1$ and $\mathrm{PC} 2$, where it is easy to see that the distribution of the five coins can be divided into four categories. The samples of coin A and coin C overlap and are then divided into one category, and the other three coins belong to three categories, respectively. This result also verifies the conclusion that the material of coin $\mathrm{A}$ and coin $\mathrm{C}$ is the same as above.

In the second way, after observing Fig. 3 and Fig. S1-S3, the spectral range from $215 \mathrm{~nm}$ to $240 \mathrm{~nm}$ with relatively more characteristic spectral lines in a small range is selected for PCA. The normalized spectral data from the above paper are used in PCA. The cumulative contribution rate $(\mathrm{PC} 1+\mathrm{PC} 2)$ is $67.404 \%$ as shown in Fig. 4a. The first three emission lines, more responsible for cluster formation, are $229.668 \mathrm{~nm}, 229.602 \mathrm{~nm}$, and 231.570 $\mathrm{nm}$. And the five coins can be better separated in Fig. $4 \mathrm{~b}$. The reason is that the coincidence degree of the spectral lines of several coins in this band is the lowest, and the elemental difference is large. Thus, the classification effect is better.

\section{Supervised analysis of five coins}

The distribution characteristics of the samples in the principal component space indicate the feasibility of classification. KNN is a simple and effective data classification method. The first two principal components of the samples and their categories are used as the training set and the labels for KNN. The samples are from five denominations of coins, so the respective denominations of the samples are used as labels. Data is standardized, KNN adopts Euclidean distance, and the number of adjacent points is 1 . Distance weight adopts equal distance. ${ }^{34}$ The classification accuracy is $81.2 \%$.

To improve the classification accuracy, the KNN model is optimized. Multiple iterations are carried out using various KNN methods. The distance measurement and distance weight of KNN are changed and repeated iterations are carried out. The distance measurement methods used include: City block, Cosine, Jaccard, Chebyshev, Spearman, Euclidean, Hamming. The number of adjacent points ranges from 1 to 128 . Distance weight includes equal distance, inverse distance and inverse distance squared. The classification accuracy of the optimized $\mathrm{KNN}$ is $84.3 \%$ by the optimized KNN. As shown in Fig. 5a, only the samples belonging to coin $\mathrm{A}$ and coin $\mathrm{C}$ were misclassified. It tends to be stable after the 14th iteration, and the minimum classification error is 0.156 in Fig. 5b. Combined with the above research, it is reasonable to believe that coin $\mathrm{A}$ and coin $\mathrm{C}$ is of the same category.

Based on this idea, the samples from coin A and coin $\mathrm{C}$ are changed into the same label. Based on the new tag, the optimized $\mathrm{KNN}$ is repeated. At this time, the classification accuracy of the optimized KNN is $100 \%$. As shown in Fig. 6, it tends to be stable after the 6th iteration, and the minimum classification error is 0 . Therefore, coin A and coin B are indeed of the same category, consistent with the previous conclusion. Thus, the classification is successful, coin A and coin C is of the same class, and each of the remaining coins is of their own class, respectively.

Coins of different denomination or material can be classified in 

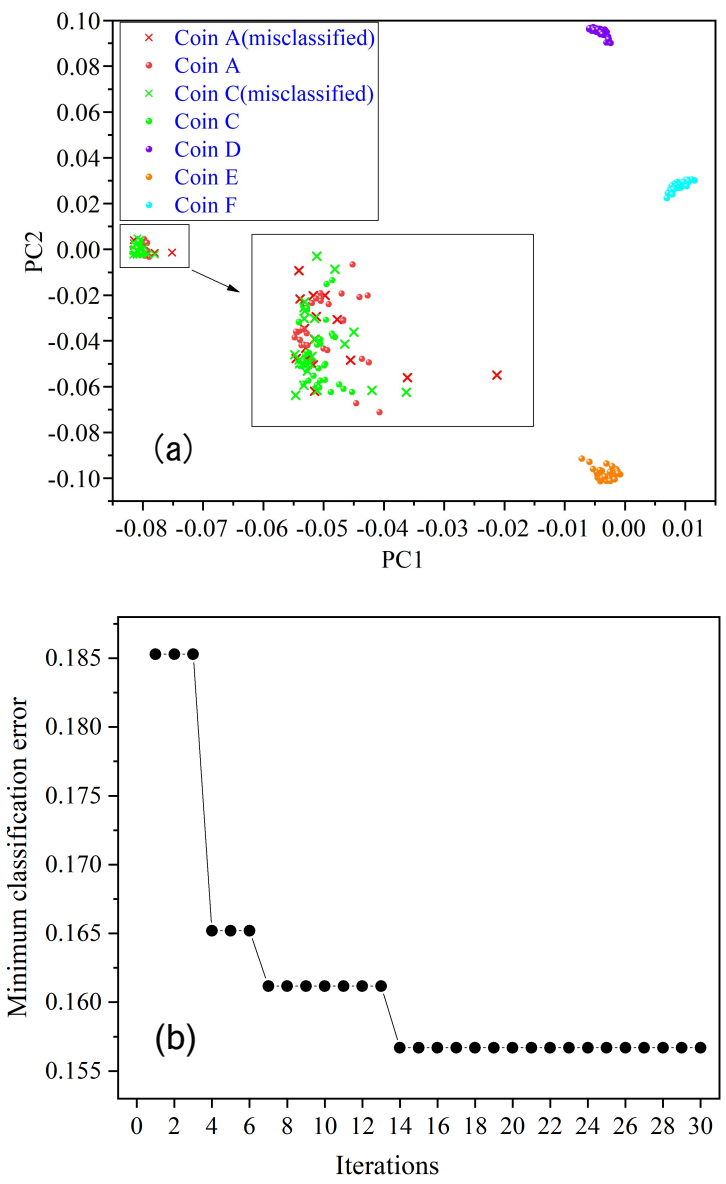

Fig. 5 (a) Scatter diagram of optimized KNN classification; (b) minimum classification error graph of optimized KNN classification.

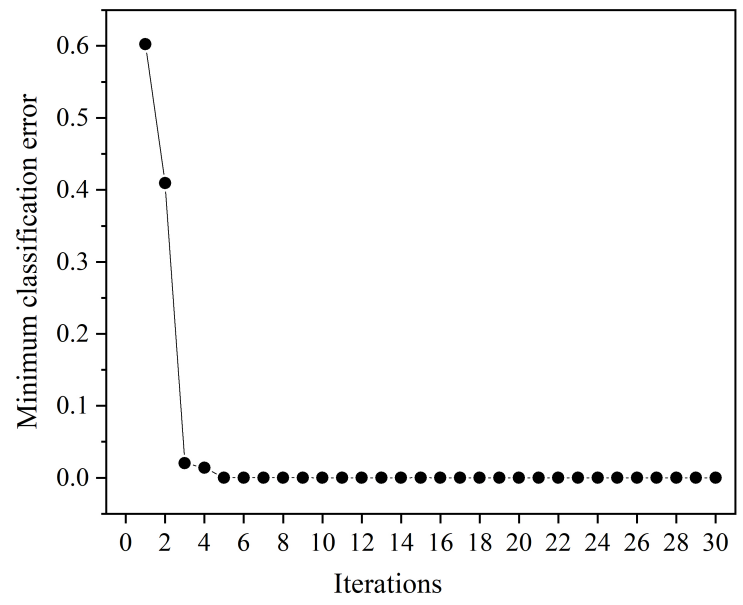

Fig. 6 Minimum classification error graph of optimized KNN classification.

this way. Of course, the classification of RMB coins in this paper is only an example based on LIBS, PCA and KNN. Coins from different countries or different ages can be detected and classified, and the type of coins can be judged more rapidly and accurately when recycling damaged coins or archaeological research.

\section{Test of the other kind of coin}

Using LIBS to detect coins rapidly, after dimensionality reduction in PCA, KNN can effectively classify different kinds of coins. To prove the feasibility of this method, samples from coin B will be used to test the process of classifying unknown coins. The spectral data of coin B will be directly used with PCA. Similarly, two ways of PCA can be used. Fig. S5a is the result of PCA from $200 \mathrm{~nm}$ to $890 \mathrm{~nm}$ and Fig. S5b is the result from $215 \mathrm{~nm}$ to $240 \mathrm{~nm}$. It can be seen that coin $\mathrm{A}$, coin $\mathrm{B}$, and coin $\mathrm{C}$ gather together. Therefore, the samples from coin $A$, coin $B$ and coin $C$ are changed into the same label. Also, the classification accuracy of the optimized $\mathrm{KNN}$ is $100 \%$ and the minimum classification error is 0 in Fig. S6. Coin $\mathrm{A}$, coin $\mathrm{B}$, and coin $\mathrm{C}$ are of the same class, and each of the remaining coins is of the same class.

To verify the above conclusion further, coin B is detected and compared with the spectrum of coin A, as shown in Fig. S7a and Fig. S7b. It is easy to see that the spectral lines of coin A and coin $\mathrm{B}$ are identical, which means that the elements contained in them are consistent, which is consistent with the classification results.

\section{Verification of LTE}

Since laser-induced plasma in the LTE state is the premise of quantitative analysis, it is necessary to verify whether plasma is in this state. ${ }^{35}$ According to the McWhirter conditions, ${ }^{36}$ the plasma in the LTE state will satisfy the following formula (Eq. 1):

$$
N_{e} \geq 1.6 \times 10^{12} T^{\frac{1}{2}}(\Delta E)^{3}
$$

$N_{e}$ is the electron number density, $T$ is the plasma temperature, and $\Delta E$ is the energy difference between the upper and the lower levels of the transitions.

In this study, $T$ is about $12496 \mathrm{~K}$, which can be calculated by the Saha-Boltzmann equation. ${ }^{37}$ Among the six characteristic spectral lines of Al $(226.309 \mathrm{~nm}, 226.837 \mathrm{~nm}, 265.202 \mathrm{~nm}$, $265.998 \mathrm{~nm} 308.157 \mathrm{~nm}$ and $309.196 \mathrm{~nm}$ ), the maximum difference between the upper and lower levels is $5.487 \mathrm{eV}$. Fig.S8 shows the spectrum line fitted with a Lorentzian curve, and it can calculate that $N_{e}$ is about $4.76 \times 10^{17} \mathrm{~cm}^{-1} \cdot{ }^{37}$ The plasma in the LTE state satisfies. ${ }^{38}$

\section{CONCLUSIONS}

Different from the existing LIBS detection and coin classification method, in this paper, five kinds of coins in circulation were successfully analyzed by LIBS, and then classified by KNN after dimension reduction by PCA which is convenient to facilitate the subsequent coin recycling work. In the case of rapid analysis, the surface of coin A (YI FEN) and coin C (WU FEN) contained the same elements, including $\mathrm{Mg}, \mathrm{Al}, \mathrm{Ca}, \mathrm{Na}$. At the same time, the AlO molecular bands were also observed. The surface of coin D 
(YI JAO) contained $\mathrm{Fe}, \mathrm{Cr}, \mathrm{Na}, \mathrm{Ca}, \mathrm{Mg}$. The surface of coin $\mathrm{E}$ (WU JIAO) contained $\mathrm{Cu}, \mathrm{Sn}, \mathrm{Na}, \mathrm{Ca}, \mathrm{Mg}$. The surface of coin $\mathrm{F}$ (YI YUAN) contained $\mathrm{Ni}, \mathrm{Na}, \mathrm{Ca}, \mathrm{Mg}$.

Two ways of PCA are used to reduce the dimension of the spectral data. In the first way (selected the spectrum range from $200 \mathrm{~nm}$ to $890 \mathrm{~nm}$ ), the cumulative contribution rate (PCA+PC2) is $65.701 \%$. In a second way (selected the spectrum range from $215 \mathrm{~nm}$ to $240 \mathrm{~nm}$ ), the cumulative contribution rate (PCA+PC2) is $67.404 \%$, and the samples can be better separated in principal component space. Based on the data of the second way, KNN was used for classification and the classification accuracy was $81.2 \%$. After optimizing KNN, the classification accuracy reached $84.3 \%$. After analyzing the scatter diagram, changing the sample label, and applying KNN again, the classification accuracy was $100 \%$. At the same time, the minimum classification error is 0 . It can be concluded that coin A and coin $\mathrm{C}$ is of the same class, and each of the remaining coins is of their own class, respectively. Further, samples of coin B were added for verification. Based on the dimensionality reduction data of PCA, the optimized KNN model was used for classification, and the classification accuracy rate was also $100 \%$. Coin B belongs to the same class as coin A and coin C. The characteristic spectral lines of $\mathrm{Al}$ were used to calculate the plasma temperature and electron number density, and the LTE state was verified.

\section{ASSOCIATED CONTENT}

Please contact the corresponding author for the Supporting Information (Fig S1-S8).

\section{AUTHOR INFORMATION}

\section{Corresponding Author}

* Y. Z. Liu

Email address: yuzhu.liu@gmail.com

\section{Notes}

The authors declare no competing financial interest.

\section{ACKNOWLEDGMENTS}

This work was supported by the National Natural Science Foundation of China (U1932149), Natural Science Foundation of Jiangsu Province (BK20191395) and Natural Science Foundation of the Higher Education Institutions of Jiangsu Province of China (18KJA140002).

\section{REFERENCES}

1. F. Kirino, N. Ohono, and S. Taguchi, J. Japan I. Met. Mater., 2019, 83, 87-96. https://doi.org/10.2320/jinstmet.J2018045

2. L. Bartoli, J. Agresti, M. Mascalchi, A. Mencaglia, I. Cacciari, and S. Siano, Quantum. Electron., 2011, 41, 663-668. https://doi.org/10.1070/QE2011v041n07ABEH014523

3. J.-N. Fang, B.-S. Yu, C.-H. Chen, D. T.-Y. Wang, and L.-P. Tan, Geoarchaeology., 2011, 26, 245-268. https://doi.org/10.1002/gea.20344

4. M. Schreiner and M. Rodrigues, Acta. Crystallogr. A., 2009, 65, S53-S53. https://doi.org/10.1107/s0108767309098973

5. E. Allen, Tribol. Lett., 2016, 64. 45 (2016). https://doi.org/10.1007/s11249-016-0780-x

6. I. G. Erusalimchik, M. N. Filippov, and I. V. Marav'eva, Prot. Met., 2005, 41, 203-204. https://doi.org/10.1007/s11124-005-0029-9

7. D. W. Hahn and N. Omenetto, Appl. Spectrosc., 2010, 64, 335A-366A. https://doi.org/10.1366/000370210793561691

8. D. W. Hahn and N. Omenetto, Appl. Spectrosc., 2012, 66, 347-419. https://doi.org/10.1366/11-06574

9. X. Wang, A. Li, N. Wazir, S. Huang, S. Guo, L. Liang, M. Zhang, B. Zou, Y. Hao, F. He, Y. Bai, W. Sun, M. Hu, and R. Liu, Opt. Express., 2018, 26, 13973-13984. https://doi.org/10.1364/oe.26.013973

10. L. B. Guo, Z. Q. Hao, M. Shen, W. Xiong, X. N. He, Z. Q. Xie, M. Gao, X. Y. Li, X. Y. Zeng, and Y. F. Lu, Opt. Express., 2013, 21, 18188-18195. https://doi.org/10.1364/oe.21.018188

11. T. A. Labutin, S. M. Zaytsev, A. M. Popov and N. B. Zorov, Opt. Express., 2014, 22, 22382-22387. https://doi.org/10.1364/oe.22.022382

12. N. Sharma, V. K. Singh, Y. Lee, S. Kumar, P. K. Rai, A. K. Pathak, and V. K. Singh, Atom. Spectrosc., 2020, 41, 234-241. https://doi.org/10.46770/as.2020.06.003

13. J. Cai, M. Dong, Y. Zhang, Y. Chen, H. Chen, Y. Liang, W. Li, and J. Lu, At. Spectrosc., 2021, 42, 43-50. https://doi.org/10.46770/as.2020.217

14. J. Hou, L. Zhang, W. Yin, S. Yao, Y. Zhao, W. Ma, L. Dong, L. Xiao and S. Jia, Opt. Express., 2017, 25, 23024-23034. https://doi.org/10.1364/oe.25.023024

15. L. F. Viana, Y. R. Suarez, C. A. Lima Cardoso, S. M. Lima, L. H. da Cunha Andrade, and S. E. Lima-Junior, Chemosphere., 2019, 228, 258-263. https://doi.org/10.1016/j.chemosphere.2019.04.070

16. M. Weidman, M. Baudelet, S. Palanco, M. Sigman, P. J. Dagdigian, and M. Richardson, Opt. Express., 2010, 18, 259-266. https://doi.org/10.1364/oe.18.000259

17. M. Wall, Z. Sun and Z. T. Alwahabi, Opt. Express., 2016, 24, 1507-1517. https://doi.org/10.1364/oe.24.001507

18. D. C. Zhang, Z. Q. Hu, Y. B. Su, B. Hai, X. L. Zhu, J. F. Zhu, and X. Ma, Opt. Express., 2018, 26, 18794-18802. https://doi.org/10.1364/oe.26.018794

19. Q. Zhang, Y. Liu, W. Yin, Y. Yan, L. Li and G. Xing, Chemosphere., 2020, 242. https://doi.org/10.1016/j.chemosphere.2019.125184

20. C. Li, Z. Hao, Z. Zou, R. Zhou, J. Li, L. Guo, X. Li, Y. Lu, and X. Zeng, Opt. Express., 2016, 24, 7850-7857. https://doi.org/10.1364/oe.24.007850

21. W. Kaplonek, T. Mikolajczyk, D. Y. Pimenov, M. K. Gupta, 
M. Mia, S. Sharma, K. Patra and M. Sutowska, Materials., 2020, 13, 19. https://doi.org/10.3390/ma13235371

22. S. Awasthi, R. Kumar, G. K. Rai and A. K. Rai, Opt. Laser. Eng., 2016, 79, 29-38. https://doi.org/10.1016/j.optlaseng.2015.11.005

23. R. Bro and A. K. Smilde, Anal. Methods-UK., 2014, 6, 2812-2831. https://doi.org/10.1039/c3ay41907j

24. D. Ballabio, F. Grisoni and R. Todeschini, Chemometr. Intell. Lab., 2018, 174, 33-44. https://doi.org/10.1016/j.chemolab.2017.12.004

25. S. Zhang, X. Li, M. Zong, X. Zhu and R. Wang, Ieee. T. Neur. Net. Lear., 2018, 29, 1774-1785. https://doi.org/10.1109/tnnls.2017.2673241

26. M.-L. Zhang and Z.-H. Zhou, Pattern. Recogn., 2007, 40, 2038-2048. https://doi.org/10.1016/j.patcog.2006.12.019

27. M. O. Bachler, M. Biscan, Z. Kregar, I. J. Badovinac, J. Dobrinic, and S. Milosevic, Spectrochim. Acta. B., 2016, 123, 163-170. https://doi.org/10.1016/j.sab.2016.08.010

28. S. Feng, X. Qiu, G. Guo, E. Zhang, Q. He, X. He, W. Ma, C. Fittschen and C. Li, Anal. Chem., 2021, 93, 4552-4558. https://doi.org/10.1021/acs.analchem.0c04995

29. X. N. He, W. Hu, C. M. Li, L. B. Guo and Y. F. Lu, Opt. Express., 2011, 19, 10997-11006. https://doi.org/10.1364/oe.19.010997
30. http://webbook.nist.gov/chemistry/form-ser/

31. Y.-T. Li, T.-A. Liu, C.-W. Chen, Y.-H. Lee and A. Yabushita, Opt. Express., 2013, 21, 21579-21586. https://doi.org/10.1364/oe.21.02157

32. L.-B. Guo, R.-F. Hao, Z.-Q. Hao, K.-H. Li, M. Shen, Z. Ren, X.-Y. Li and X.-Y. Zeng, Acta. Phys. Sin-ch. Ed., 2013, 62. https://doi.org/10.7498/aps.62.224211

33. Y. Zhangcheng, Y. Liu, S. Saleem, Q. Zhang, Y. Chen, Y. Qu, and X. Lu, J. Laser. Appl., 2020, 32. https://doi.org/10.2351/7.0000137

34. D. Ballabio, F. Grisoni, and R. Todeschini, Chemometr. Intell. Lab., 2018, 174, 33-44. https://doi.org/10.1016/j.chemolab.2017.12.004

35. X. Lu, Y. Liu, Q. Zhang, and L. Li, Laser. Phys. Lett., 2020, 17. https://doi.org/10.1088/1612-202X/ab5c23

36. A. A. Khedr, M. A. Sliem and M. Abdel-Harith, Appl. Spectrosc., 2020, https://doi.org/10.1177/0003702820973040

37. A. M. El Sherbini, T. El Sherbini, H. Hegazy, G. Cristoforetti, S. Legnaioli, L. Pardini, V. Palleschi, A. Salvetti, and E. Tognoni, Spectrosc. Lett., 2007, 40, 643-658. https://doi.org/10.1080/00387010701300958

38. M. Habibpour, P. Parvin, and R. Amrollahi, Appl. Optics, 2021, 60, 1099-1109. https://doi.org/10.1364/ao.416032 